\title{
On a Fredholm-Volterra integral equation
}

\author{
Alexandru-Darius Filip and Ioan A. Rus
}

Abstract. In this paper we give conditions in which the integral equation

$$
x(t)=\int_{a}^{c} K(t, s, x(s)) d s+\int_{a}^{t} H(t, s, x(s)) d s+g(t), t \in[a, b],
$$

where $a<c<b, K \in C([a, b] \times[a, c] \times \mathbb{B}, \mathbb{B}), H \in C([a, b] \times[a, b] \times \mathbb{B}, \mathbb{B})$, $g \in C([a, b], \mathbb{B})$, with $\mathbb{B}$ a (real or complex) Banach space, has a unique solution in $C([a, b], \mathbb{B})$. An iterative algorithm for this equation is also given.

Mathematics Subject Classification (2010): 45N05, 47H10, 47H09, 54H25.

Keywords: Fredholm-Volterra integral equation, existence, uniqueness, contraction, fiber contraction, Maia theorem, successive approximation, fixed point, Picard operator.

\section{Introduction}

The following type of integral equation was studied by several authors (see [11], $[2],[3],[6],[1],[5],[10],[7], \ldots)$,

$$
x(t)=\int_{a}^{c} K(t, s, x(s)) d s+\int_{a}^{t} H(t, s, x(s)) d s+g(t), t \in[a, b],
$$

where $a<c<b, K \in C([a, b] \times[a, c] \times \mathbb{B}, \mathbb{B}), H \in C([a, b] \times[a, b] \times \mathbb{B}, \mathbb{B}), g \in C([a, b], \mathbb{B})$, with $(\mathbb{B},|\cdot|)$ a (real or complex) Banach space.

The aim of this paper is to give some conditions on $K$ and $H$ in which the equation (1.1) has a unique solution in $C([a, b], \mathbb{B})$. To do this, we shall use the contraction principle, the fiber contraction principle ([9], [13], [10], [11]) and a variant of Maia fixed point theorem given in [8] (see also [4]).

\section{Preliminaries}

Let us recall some notions, notations and fixed point results which will be used in this paper. 


\subsection{Picard operators and weakly Picard operators}

Let $(X, \rightarrow)$ be an $L$-space $((X, d), \stackrel{d}{\rightarrow} ;(X, \tau), \stackrel{\tau}{\rightarrow} ;(X,\|\cdot\|), \stackrel{\|\cdot\|}{\rightarrow}, \rightarrow ; \ldots)$. An operator $A:(X, \rightarrow) \rightarrow(X, \rightarrow)$ is called weakly Picard operator (WPO) if the sequence $\left(A^{n}(x)\right)_{n \in \mathbb{N}}$ converges for all $x \in X$ and the limit (which generally depends on $x$ ) is a fixed point of $A$.

If an operator $A$ is $W P O$ and the fixed point set of $A$ is a singleton, i.e.,

$$
F_{A}=\left\{x^{*}\right\}
$$

then, by definition, $A$ is called Picard operator $(P O)$.

For a $W P O, A:(X, \rightarrow) \rightarrow(X, \rightarrow)$, we define the limit operator $A^{\infty}:(X, \rightarrow) \rightarrow$ $(X, \rightarrow)$, by $A^{\infty}(x):=\lim _{n \rightarrow \infty} A^{n}(x)$. We remark that, $A^{\infty}(X)=F_{A}$, i.e., $A^{\infty}$ is a set retraction of $X$ on $F_{A}$.

\subsection{Fiber contraction principle}

Regarding this principle, some important results were given in [12] and [13].

Fiber Contraction Theorem. Let $(X, \rightarrow)$ be an L-space, $(Y, d)$ be a metric space, $B: X \rightarrow X, C: X \times Y \rightarrow Y$ and $A: X \times Y \rightarrow X \times Y, A(x, y):=(B(x), C(x, y))$. We suppose that:

(i) $(Y, d)$ is a complete metric space;

(ii) $B$ is a WPO;

(iii) $C(x, \cdot): Y \rightarrow Y$ is an l-contraction, for all $x \in X$;

(iv) $C: X \times Y \rightarrow Y$ is continuous.

Then $A$ is a WPO. Moreover, if $B$ is a PO, then $A$ is a PO.

Generalized Fiber Contraction Theorem. Let $(X, \rightarrow)$ be an L-space and $\left(X_{i}, d_{i}\right), i=$ $\overline{1, m}, m \geq 1$ be metric spaces. Let $A_{i}: X_{0} \times \ldots \times X_{i} \rightarrow X_{i}, i=\overline{0, m}$, be some operators. We suppose that:

(i) $\left(X_{i}, d_{i}\right), i=\overline{1, m}$, are complete metric spaces;

(ii) $A_{0}$ is a WPO;

(iii) $A_{i}\left(x_{0}, \ldots, x_{i-1}, \cdot\right): X_{i} \rightarrow X_{i}, i=\overline{1, m}$, are $l_{i}$-contractions;

(iv) $A_{i}, i=\overline{1, m}$, are continuous.

Then the operator $A: X_{0} \times \ldots \times X_{m} \rightarrow X_{0} \times \ldots \times X_{m}$, defined by

$$
A\left(x_{0}, \ldots, x_{m}\right):=\left(A_{0}\left(x_{0}\right), A_{1}\left(x_{0}, x_{1}\right), \ldots, A_{m}\left(x_{0}, \ldots, x_{m}\right)\right)
$$

is a WPO. Moreover, if $A_{0}$ is a PO, then $A$ is a PO.

\subsection{A variant of Maia fixed point theorem}

We recall here the following variant of Maia fixed point theorem, given by I.A. Rus in [8]:

Theorem 2.1. Let $X$ be a nonempty set, $d$ and $\rho$ be two metrics on $X$ and $A: X \rightarrow X$ be an operator. We suppose that:

(1) there exists $c>0$ such that $d(A(x), A(y)) \leq c \rho(x, y)$, for all $x, y \in X$;

(2) $(X, d)$ is a complete metric space;

(3) $A:(X, d) \rightarrow(X, d)$ is continuous; 
(4) $A:(X, \rho) \rightarrow(X, \rho)$ is an l-contraction.

Then:

(i) $F_{A}=\left\{x^{*}\right\}$;

(ii) $A:(X, d) \rightarrow(X, d)$ is $P O$.

\section{Operatorial point of view on equation (1.1)}

Let $X:=C([a, b], \mathbb{B})$ and $T: X \rightarrow X$ be defined by

$$
T(x)(t):=\int_{a}^{c} K(t, s, x(s)) d s+\int_{a}^{t} H(t, s, x(s)) d s+g(t), t \in[a, b] .
$$

For $x \in X$, we denote by $u:=\left.x\right|_{[a, c]}$ and $v:=\left.x\right|_{[c, b]}$. If $x$ is a solution of the equation (1.1) (i.e. a fixed point of $T$ ), then

$$
u(t)=\int_{a}^{c} K(t, s, u(s)) d s+\int_{a}^{t} H(t, s, u(s)) d s+g(t), t \in[a, c]
$$

and

$$
\begin{aligned}
v(t) & =\int_{a}^{c} K(t, s, u(s)) d s+\int_{a}^{c} H(t, s, u(s)) d s \\
& +\int_{c}^{t} H(t, s, v(s)) d s+g(t), t \in[c, b] .
\end{aligned}
$$

Let $X_{1}:=C([a, c], \mathbb{B}), X_{2}:=C([c, b], \mathbb{B})$ and

$T_{1}: X_{1} \rightarrow X_{1}, T_{1}(u)(t):=$ the second part of $(3.1)$,

$T_{2}: X_{1} \times X_{2} \rightarrow X_{2}, T_{2}(u, v)(t):=$ the second part of (3.2).

The mappings $T_{1}$ and $T_{2}$ allow us to construct the triangular operator

$$
\tilde{T}: X_{1} \times X_{2} \rightarrow X_{1} \times X_{2}, \tilde{T}(u, v):=\left(T_{1}(u), T_{2}(u, v)\right) \text {, for all }(u, v) \in X_{1} \times X_{2} .
$$

Remark 3.1. If $\left(u^{*}, v^{*}\right) \in F_{\tilde{T}}$, then $u^{*}(c)=v^{*}(c)$. So the function $x^{*} \in X$, defined by

$$
x^{*}(t):=\left\{\begin{array}{l}
u^{*}(t), t \in[a, c] \\
v^{*}(t), t \in[c, b]
\end{array}\right.
$$

is a fixed point of $T$, i.e., a solution of (1.1).

Remark 3.2. For $\left(u_{0}, v_{0}\right) \in X_{1} \times X_{2}$ we consider the successive approximations corresponding to the operator $\tilde{T},\left(u_{n+1}, v_{n+1}\right)=\tilde{T}\left(u_{n}, v_{n}\right), n \in \mathbb{N}$. We observe that, for $n \in \mathbb{N}^{*}, u_{n}(c)=v_{n}(c)$. So, the function $x_{n}$, defined by

$$
x_{n}(t):=\left\{\begin{array}{l}
u_{n}(t), t \in[a, c] \\
v_{n}(t), t \in[c, b]
\end{array}\right.
$$

is in $X$. 
Remark 3.3. Let $Y \subset X_{1} \times X_{2}$ be defined by

$$
Y:=\left\{(u, v) \in X_{1} \times X_{2} \mid u(c)=v(c)\right\} .
$$

The operator $R: X \rightarrow Y$, defined by $R(x):=\left(\left.x\right|_{[a, c]},\left.x\right|_{[c, b]}\right)$ is a bijection. From the above definitions, it is clear that $T(x)=\left(R^{-1} \tilde{T} R\right)(x)$ and the $n^{\text {th }}$ iterate of $T$ is $T^{n}=R^{-1} \tilde{T}^{n} R$.

In conclusion, to study the equation (1.1) (which is equivalent with $x=T(x)$ ) it is sufficient to study the fixed point of the operator $\tilde{T}$. If $\left(u^{*}, v^{*}\right) \in F_{\tilde{T}}$ then $R^{-1}\left(u^{*}, v^{*}\right) \in F_{T}$.

\section{Existence and uniqueness of solution of equation (1.1)}

In what follows, in addition to the continuity of $H, K$ and $g$, we suppose on $K$ and $H$ that:

(i) There exists $L_{1} \in C([a, b] \times[a, c], \mathbb{B})$ such that:

$|K(t, s, \xi)-K(t, s, \eta)| \leq L_{1}(t, s)|\xi-\eta|$, for all $t \in[a, b], s \in[a, c], \xi, \eta \in \mathbb{B}$.

(ii) There exists $L_{2} \in C([a, b] \times[a, b], \mathbb{B})$ such that:

$$
|H(t, s, \xi)-H(t, s, \eta)| \leq L_{2}(t, s)|\xi-\eta|, \text { for all } t, s \in[a, b], \xi, \eta \in \mathbb{B} .
$$

(iii) $\left(\int_{[a, c] \times[a, c]}\left(L_{1}(t, s)+L_{2}(t, s)\right)^{2} d t d s\right)^{\frac{1}{2}}<1$.

The basic result of our paper is the following.

Theorem 4.1. In the above conditions we have that:

(1) The equation $(1.1)$ has in $C([a, b], \mathbb{B})$ a unique solution $x^{*}$.

(2) The operator $\tilde{T}$ is a Picard operator with respect to $\stackrel{\text { unif. }}{\rightarrow}$. Let $F_{\tilde{T}}=\left\{\left(u^{*}, v^{*}\right)\right\}$.

(3) The operator $T$ is a Picard operator with respect to $\stackrel{\text { unif. }}{\rightarrow}$ and $F_{T}=\left\{x^{*}\right\}$. Moreover, $x^{*}=R^{-1}\left(u^{*}, v^{*}\right)$.

Proof. From the remarks which were given in $\S 3$, it is sufficient to prove that the operator $\tilde{T}$ is a Picard operator with respect to the uniform convergence on $X_{1} \times X_{2}$.

In order to apply the Fiber contraction principle, we shall prove that:

(j) $T_{1}:\left(X_{1}, \stackrel{\text { unif. }}{\rightarrow}\right) \rightarrow\left(X_{1}, \stackrel{\text { unif. }}{\rightarrow}\right)$ is a Picard operator;

(jj) $T_{2}(u, \cdot):\left(X_{2},\|\cdot\|_{\tau}\right) \rightarrow\left(X_{2},\|\cdot\|_{\tau}\right)$ is a contraction.

Let us prove $(j)$.

We consider on $X_{1}$, the norms $\|\cdot\|_{\infty}$ and $\|\cdot\|_{L^{2}}$. By using the assumptions $(i)$ and $(i i)$, we have the following estimations:

$$
\begin{aligned}
\left|T_{1}\left(u_{1}\right)(t)-T_{1}\left(u_{2}\right)(t)\right| & \leq \int_{a}^{c}\left|K\left(t, s, u_{1}(s)\right)-K\left(t, s, u_{2}(s)\right)\right| d s \\
& +\int_{a}^{t}\left|H\left(t, s, u_{1}(s)\right)-H\left(t, s, u_{2}(s)\right)\right| d s
\end{aligned}
$$




$$
\begin{aligned}
& \leq \int_{a}^{c} L_{1}(t, s)\left|u_{1}(s)-u_{2}(s)\right| d s+\int_{a}^{c} L_{2}(t, s)\left|u_{1}(s)-u_{2}(s)\right| d s \\
& \stackrel{\text { Hölder's }}{\text { inequality }}\left(\int_{a}^{c} L_{1}(t, s)^{2} d s\right)^{\frac{1}{2}}\left(\int_{a}^{c}\left|u_{1}(s)-u_{2}(s)\right|^{2} d s\right)^{\frac{1}{2}} \\
& +\left(\int_{a}^{c} L_{2}(t, s)^{2} d s\right)^{\frac{1}{2}}\left(\int_{a}^{c}\left|u_{1}(s)-u_{2}(s)\right|^{2} d s\right)^{\frac{1}{2}} \cdot
\end{aligned}
$$

By taking the $\max _{t \in[a, c]}$ in the above inequalities, there exists a real positive constant

$$
c:=\max _{t \in[a, c]}\left(\int_{a}^{c} L_{1}(t, s)^{2} d s\right)^{\frac{1}{2}}+\max _{t \in[a, c]}\left(\int_{a}^{c} L_{2}(t, s)^{2} d s\right)^{\frac{1}{2}}
$$

such that

$$
\left\|T_{1}\left(u_{1}\right)-T_{1}\left(u_{2}\right)\right\|_{\infty} \leq c\left\|u_{1}-u_{2}\right\|_{L^{2}}, \text { for all } u_{1}, u_{2} \in X_{1} .
$$

On the other hand, we have that

$$
\begin{aligned}
\left\|T_{1}\left(u_{1}\right)-T_{1}\left(u_{2}\right)\right\|_{L^{2}} & =\left(\int_{a}^{c}\left|T_{1}\left(u_{1}\right)(t)-T_{1}\left(u_{2}\right)(t)\right|^{2} d t\right)^{\frac{1}{2}} \\
& \leq\left(\int_{a}^{c}\left(\int_{a}^{c}\left(L_{1}(t, s) d s+L_{2}(t, s)\right)^{2} d s\right)\left\|u_{1}-u_{2}\right\|_{L^{2}}^{2} d t\right)^{\frac{1}{2}} \\
& =\left(\int_{a}^{c} \int_{a}^{c}\left(L_{1}(t, s)+L_{2}(t, s)\right)^{2} d s d t\right)^{\frac{1}{2}}\left\|u_{1}-u_{2}\right\|_{L^{2}},
\end{aligned}
$$

for all $u_{1}, u_{2} \in X_{1}$.

By using the assumption (iii), it follows that the operator $T_{1}$ is a contraction with respect to $\|\cdot\|_{L^{2}}$ on $X_{1}$.

The conclusion follows from the variant of Maia theorem.

Let us prove $(j j)$.

For $t \in[c, b]$ and $M_{L_{2}}:=\max _{t, s \in[c, b]} L_{2}(t, s)$, we have that

$$
\begin{aligned}
\left|T_{2}\left(u, v_{1}\right)(t)-T_{2}\left(u, v_{2}\right)(t)\right| & \leq \int_{c}^{t}\left|H\left(t, s, v_{1}(s)\right)-H\left(t, s, v_{2}(s)\right)\right| d s \\
& \leq \int_{c}^{t} L_{2}(t, s)\left|v_{1}(s)-v_{2}(s)\right| d s \\
& \leq M_{L_{2}} \int_{c}^{t}\left|v_{1}(s)-v_{2}(s)\right| e^{-\tau(s-c)} e^{\tau(s-c)} d s \\
& \leq M_{L_{2}}\left\|v_{1}-v_{2}\right\|_{\tau} \int_{c}^{t} e^{\tau(s-c)} d s \leq M_{L_{2}}\left\|v_{1}-v_{2}\right\|_{\tau} \frac{e^{\tau(t-c)}}{\tau} .
\end{aligned}
$$

It follows that

$$
\left|T_{2}\left(u, v_{1}\right)(t)-T_{2}\left(u, v_{2}\right)(t)\right| e^{-\tau(t-c)} \leq \frac{M_{L_{2}}}{\tau}\left\|v_{1}-v_{2}\right\|_{\tau} .
$$


By taking $\max _{t \in[c, b]}$ and by choosing $\tau>M_{L_{2}}$, there exists a real positive constant

$$
l:=\frac{M_{L_{2}}}{\tau}<1
$$

such that

$$
\left\|T_{2}\left(u, v_{1}\right)-T_{2}\left(u, v_{2}\right)\right\|_{\tau} \leq l\left\|v_{1}-v_{2}\right\|_{\tau}, \text { for all } v_{1}, v_{2} \in X_{2} .
$$

Remark 4.2. Let $\mathbb{K}:=\mathbb{R}$ or $\mathbb{C},|\cdot|$ be a norm on $\mathbb{B}:=\mathbb{K}^{m}\left(|\cdot|_{1},|\cdot|_{2},|\cdot|_{\infty}, \ldots\right)$, $a<c<b, K=\left(K_{1}, \ldots, K_{m}\right) \in C\left([a, b], \mathbb{K}^{m}\right)$ and $H=\left(H_{1}, \ldots, H_{m}\right) \in C\left([a, b], \mathbb{R}^{m}\right)$. In this case, the equation (1.1) takes the following form

$$
\left\{\begin{aligned}
x_{1}(t) & =\int_{a}^{c} K_{1}\left(t, s, x_{1}(s), \ldots, x_{m}(s)\right) d s \\
& +\int_{a}^{t} H_{1}\left(t, s, x_{1}(s), \ldots, x_{m}(s)\right) d s, t \in[a, b] \\
& \vdots \\
x_{m}(t) & =\int_{a}^{c} K_{m}\left(t, s, x_{1}(s), \ldots, x_{m}(s)\right) d s \\
& \int_{a}^{t} H_{m}\left(t, s, x_{1}(s), \ldots, x_{m}(s)\right) d s, t \in[a, b] .
\end{aligned}\right.
$$

From Theorem 4.1 we have an existence and uniqueness result for the system (4.1).

In the case when $\mathbb{B}$ is a Banach space of infinite sequences with elements in $\mathbb{K}$ $\left(c(\mathbb{K}), C_{p}(\mathbb{K}), m(\mathbb{K}), l^{p}(\mathbb{K}), \ldots\right)$ we have from Theorem 4.1 an existence and uniqueness result for an infinite system of Fredholm-Volterra integral equations.

\section{References}

[1] Bolojan, O.-M., Fixed Point Methods for Nonlinear Differential Systems with Nonlocal Conditions, Casa Cărţii de Ştiinţă, Cluj-Napoca, 2013.

[2] Boucherif, A., Differential equations with nonlocal boundary conditions, Nonlinear Anal., 47(2001), 2419-2430.

[3] Boucherif, A., Precup, R., On the nonlocal initial value problem for first order differential equations, Fixed Point Theory, 4(2003), 205-212.

[4] Filip, A.-D., Fixed Point Theory in Kasahara Spaces, Casa Cărţii de Ştiinţă, ClujNapoca, 2015.

[5] Nica, O., Nonlocal initial value problems for first order differential systems, Fixed Point Theory, 13(2012), 603-612.

[6] Petruşel, A., Rus, I.A., A class of functional integral equations with applications to a bilocal problem, 609-631. In: Topics in Mathematical Analysis and Applications (Rassias, Th.M. and Tóth, L., Eds.), Springer, 2014.

[7] Precup, R., Methods in Nonlinear Integral Equations, Kluwer, Dordrecht-BostonLondon, 2002.

[8] Rus, I.A., On a fixed point theorem of Maia, Stud. Univ. Babeş-Bolyai Math., 22(1977), no.. 1, 40-42. 
[9] Rus, I.A., Picard operators and applications, Sci. Math. Jpn., 58(2003), 191-219.

[10] Rus, I.A., Abstract models of step method which imply the convergence of successive approximations, Fixed Point Theory, 9(2008), no. 1, 293-307.

[11] Rus, I.A., Some variants of contraction principle in the case of operators with Volterra property: step by step contraction principle, Adv. Theory Nonlinear Anal. Appl., 3(2019), no. $3,111-120$.

[12] Rus, I.A., Şerban, M.-A., Basic problems of the metric fixed point theory and the relevance of a metric fixed point theorem, Carpathian J. Math., 29(2013), no. 2, 239-258.

[13] Şerban, M.-A., Teoria Punctului Fix pentru Operatori Definiţi pe Produs Cartezian, Presa Univ. Clujeană, Cluj-Napoca, 2002.

Alexandru-Darius Filip

Babeş-Bolyai University,

Faculty of Economics and Business Administration,

Department of Statistics-Forecasts-Mathematics,

Teodor Mihali Street, No. 58-60,

400591 Cluj-Napoca, Romania

e-mail: darius.filip@econ.ubbcluj.ro

Ioan A. Rus

Babeş-Bolyai University,

Faculty of Mathematics and Computer Sciences,

1, Kogălniceanu Street,

400084 Cluj-Napoca, Romania

e-mail: iarus@math.ubbcluj.ro 